\title{
Conceptual Model and System for Genre-Focused Interactive Storytelling
}

\author{
Börje F. Karlsson ${ }^{1}$ and Antonio L. Furtado ${ }^{2}$ \\ ${ }^{1}$ Microsoft Research, Beijing, China \\ ${ }^{2}$ Departamento de Informática, PUC-Rio, Rio de Janeiro, RJ, Brazil \\ borjekar@microsoft.com, furtado@inf.puc-rio.br
}

\begin{abstract}
This paper describes a conceptual model for the definition of a genre in the context of Interactive Storytelling and its implementation in LogTell-R, a system for the interactive creation of stories. This work builds on a previous system and experiments with plan recognition and discusses the foundations of our model to allow the creation of varied and coherent stories within a genre.
\end{abstract}

Keywords: storytelling, conceptual model, genre, plan generation / recognition.

\section{Introduction}

Schank [17] writes that humans understand the world in terms of stories. Arguably, the human brain has a natural affinity not only for enjoying narratives, but also for creating them [19]. Thus, the dynamic and interactive generation of stories is an interesting problem. However, the generation of stories that are coherent and interesting, while accounting for authors, and audience interactions remains an unsettled issue. A wide range of approaches has tried to integrate storytelling and interactive entertainment (narrative and gameplay), with limited success, mostly by overly restricting user interaction options. While this may be a pragmatic attempt to simplify the problem space, we feel that more generative approaches are required if the field is to become truly successful. One of the main open problems in the generative craft of stories resides in how stories may be generated and told. While it is probably not possible to define an ideal general model for good stories, we claim that sound methods to organize and combine events must be considered to confer enough dramatic power to narratives. Some approaches do make use of special narrative functions that enforce narrative principles in the context of the whole story (like raising tension, introducing dilemmas, or some mechanism to "move the story forward") [10], but while these efforts improve user engagement they still do not guarantee diversity of experience. A possible avenue for the development of more generative systems that can create interesting stories is related to the definition of a genre. Once a genre is specified with some rigour in a constructive way, it becomes possible not only to determine whether a given plot is a legitimate representative of the genre, but also to generate such plots. To tackle the problem of creating a more generative model to support the production of stories, we draw on previous 
system-building experience and on the four master tropes identified by semiotic research, namely: metaphor, metonymy synecdoche, and irony (which are the basic rhetorical structures by which we make sense of experience [2, 5]). By offering mechanisms derived from event relations connected to these tropes, we intend to augment the expressiveness of the narrative model.

This paper describes a conceptual model used to represent plots in a given genre and presents extensions to better support event relations and enhance the space of possibly interesting stories in the context of a plot generation system. We outline how an intended genre (to whose conventions the plots must conform) can be modelled and describe LogTell-R [10], an extended version of LOGTELL [4]. This new system uses plan recognition and plan generation in the creation of stories and a plot algebra to help define the story space. We then show how the new system works on the basis of the identified event relations, also indicating what is done to narrow the gap between the conceptual model and the implementation while helping to construct the possible plots. Finally, we offer some remarks on the results obtained.

\section{Story Generation Systems}

Story generation systems (SGSs) employ different strategies to configure their story models, which can be broadly classified as character-based, plot-based, and userexperience models. In character-based models, the storyline results from the interaction among virtual agents. Their main advantage is the ability for anytime user intervention, but, while powerful in terms of interaction, such interference level may lead the plot to uncharacteristic situations. Additionally, there is no guarantee that narratives emerging from the interaction between agents will create interesting drama. By contrast, in plot-based models, characters should follow more rigid rules, specifying the intended plot structure. User intervention is thus more limited, but it is usually easier to guarantee coherence and a measure of dramatic power. Lastly, SGSs with user-experience models, focus on the user by addressing individual preferences via user profiling and measuring the interestingness of story pieces.

We feel that an approach that fits in between plot-based and character-based is the best option, given that plots and characters are interlocking elements that cannot exist without each other [7]. Specifically, we try to conciliate both. User-experience concerns can be dealt with at later stages; as long as we can guarantee that enough variety of stories can be generated, possibly while considering changes in dramatization / presentation of story events. Even though it is argued that the key aspect of an interactive narrative is the story representation used to encode the author's vision of the experiences (story space [12]), little attention is given to what qualities such space should have. Most approaches to plot-influenced storytelling models consider only stories formed by sequences of events using their inherent temporal characteristics or simple causality relations, which are clearly not enough to guarantee interesting and varied stories. Other approaches use dilemma-inducing or tension-raising events, paying no attention to the relations between events in the plot model. Few efforts dedicate enough attention to relations between events. Three 
relevant exceptions are [15], [11], and [14]. Pinhanez [15] deals with a temporal model of events, providing mechanisms to handle their interlacing temporal relations. Building on the same principle, the Joseph system [11] claims to provide a formal framework to relate story components to one another. In it, a story is modelled as having two sub-components, a setting and an episode list, both of which have temporal intervals associated with them and information about event relationships is specified in its rules. The Joseph system is (to our knowledge) the first system constructed from an explicit, formal model for stories. ISRST argues that relations determined by the rhetorical context of events is key [14] and introduce an ontology model based on the organization of events. In ISRST, a relation is a binding between two entities, which refers to a specific rhetorical function. They further claim that "its use is impractical for the purpose of content creation, since most human beings make use of a more limited set of relations to construct and remember stories" [14]. To match this limitation, a reduced set of nine relations is considered. However, their ideas are not implemented in a concrete system.

Lastly, content creation tools in place to make the underlying complexity of the knowledge base transparent to authors is paramount, especially if the representation uses complex models or specialized programming languages. For example, although Façade has been a successful experiment, its architecture requires a great effort from authors. It uses four different content languages and took two years to author a game with only one scene, two characters, and that takes about 20 minutes to complete [13].

\section{The LOGTELL Plot Composition Model}

In summary, LOGTELL is a logic-based tool for the interactive generation and dramatization of stories via the use of a plan-generation system. The main difference between its conceptual model and similar planning systems is that it does not assume the existence of one goal for the story as a whole. Instead, at the beginning of the plot (and after each planning phase), goal-inference rules are used to consider new goals induced for the various characters. Its design borrows notions from narratology, in particular, the distinction of three levels in literary composition: fabula, story, and text [1]. At the fabula level, the characters in the narrative are introduced, as well as the narrative plot - corresponding to a chronological sequence of events. The story level concerns a different representation specific to these events and how they are narrated to an audience, be it through a temporal re-ordering of the events or other narrative techniques. The last level relates to the medium used to tell the story, such as natural language, movie, or any other communication medium.

When breaking down the IS problem into its smaller sub-parts, a similar separation is observed between the story generation and narration phases [10]. In SGSs, this breakdown means that different modules handle the two parts. In discussing the conceptual model behind the story space in LOGTELL, we are interested only in the fabula level, within its Plot Manager, where the narrative plot being manipulated consists of a partially-ordered set of events. Here we should recall that plot managers, in general, are interactive story generators that receive as input an already created 
piece of story (initially empty) and present as output a suggestion on how to continue the story. Their main function is to effect corrections so the story plot can develop correctly. If some inconsistency is detected in the input, the manager intervenes in order to ensure that the original goal can be reached, satisfying all the necessary constraints.

To model a chosen environment to which the plots to be composed should belong, it is necessary to specify at least: a) what can exist at some state of the underlying world; b) how states can be changed; and c) the factors driving the characters to act.

Accordingly, LOGTELL defines three schemas: static, dynamic and behavioural, representing, respectively: a) Storyworld: the world description takes the form of a set of facts (state), introducing the characters and their initial situation (relationships and attributes); b) World State Changes: narratives are composed of events and an event is a transition from a valid world state to another, which should also be valid; and c) Character's behaviour: to model the reasons for each character's actions, LOGTELL uses goal-inference rules specifying (in a logic formalism) the motivations of these agents when certain situations occur.

State transitions consist of a limited repertoire of pre-defined domain-oriented operations. A similar notion has been proposed by Vladimir Propp [16]. LOGTELL equates the notion of event with the state-change brought about by the execution of an operation by some agent. The dynamic schema is thus composed of a repertoire of pre-defined operations (typical of the chosen genre) in which characters can take part.

The generation of a plot starts by inferring goals for the characters from the initial configuration. A planner inserts events in the plot in order to allow the characters to try to fulfill their goals. The result is then presented to the user. If the user accepts it, the process continues by inferring new goals. The process alternates goal-inference and plan generation until the user decides to stop or no new goal is inferred.

To further explore the possibilities of generating varied coherent stories in the given "fairy-tale genre", we enabled mechanisms also for plan-recognition. Experiments with the system demonstrate that combining plan-recognition/generation and user participation constitutes a promising strategy towards the production of plots which are both diverse and coherent, as it helps guide the user [8]. However, that does not guarantee dramatic power. Sound methods to combine events must be considered to accomplish this goal; which led to the necessity to further extend the utilized model. We do not claim that the present effort is sufficient to create a fully immersive experience. We endeavour, as a more limited objective, to explore the possibilities of generating a large variety of stories by applying this strategy over a given genre.

\section{Event Relations in Plot Composition}

Many narratologists agree that the most important relation between events is the causal one. Nevertheless, this is not the only relation taken into consideration when human beings try to create story patterns in their minds [14]. The impact on the audience very much depends on how events are combined. Furtado [6] suggests that at least four concerns are involved in plot composition: a) the plot must be formed by 
a coherent sequence of events; b) for each position in the sequence, several alternative choices should apply; c) non-trivial interesting sequences must permit unexpected shifts along the way; and d) one may need to go down to details to better visualize the events or, conversely, to summarize detailed event sequences. These concerns have led to the identification of four relations between pairs of events that play a basic role in a story, and to the introduction of a fourfold perspective of plot composition in the context of IS. The four relations are: a) syntagmatic - the occurrence of the first leaves the world in a state wherein the occurrence of the second is coherent. The syntagmatic relation between events induces a weak form of causality or enablement, which justifies their sequential ordering inside the plot; b) paradigmatic - the events can be seen as alternative ways to accomplish a similar kind of higher-level action, so that both achieve the same basic effect in a significantly different way; c) antithetic the events oppose or contradict each other, since the occurrence of each of them implies a radically different context; and d) meronymic - referring to the decomposition of an event into lower-level events, thus providing a more detailed account of the action on hand.

The first two relations were already handled in LOGTELL. To extend its functionality, we included the use of a hierarchy of (generalized) events and planrecognition mechanisms during the plot composition phase [8]. This hierarchy consists of a conveniently structured library containing sequences of events with partial order relationships between them, which can be adapted if necessary to specific circumstances. The typical plans (also called complex operations) stored in the library result from meronymic relations between other operations (Proppinspired). Complex operations formed by generalization are also represented, branching down to specialized operations corresponding to alternative ways to reach the same main effects.

Transgressive plots based on antithetic relations can add a dramatic impact, by introducing unexpected turns in the narrative, e.g. by the sudden recognition of a wrong belief, or by a radical change of fortune (Aristotle's "discovery" and "reversal"). If his beloved princess is abducted, the hero would normally rescue her but the antithetically related capture event would be justified if she unexpectedly falls in love with the captor (the so-called Stockholm syndrome). Mechanisms to support this kind of dramatic change must be provided, one of the possibilities being to allow arbitrary user interventions at certain points (recalling the deus ex-machina device).

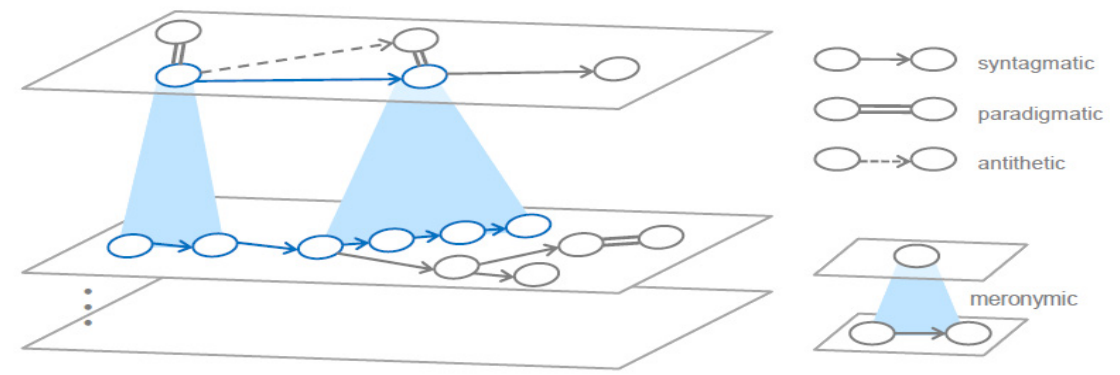

Fig. 1. Relations between events in the story space [6] 


\subsection{Genre as Story Space}

Finding an answer to the question of what constitutes a given literary genre should allow one to determine whether or not a story can be classified as belonging to it. However proving that a conceptual model $\mathbf{M}$ for specifying a genre $\mathbf{G}$, formulated as discussed in this paper, can fully capture how the genre is understood by a literary expert can be an over-ambitious effort. Yet, a useful approximation is attainable, taking the form of a definition by extension. If a system can generate stories according to the specified conceptual model $\mathbf{M}$, the space of plots that can be generated might be regarded as constituting a genre $\mathbf{G}^{\mathbf{M}}$ defined by the model, where plot has the usual meaning of sequence of events. It would be left to the designer of $\mathbf{M}$ the task of refining the model specification in order to achieve increasingly closer matches between the intuitive view of $\mathbf{G}$ and $\mathbf{G}^{\mathbf{M}}$. This is basically the role of the presented conceptual model. As argued, it determines the genre of the stories to be generated in correspondence to the story space of a system that uses that model. Such definition of genre as the set of stories that can be generated from a model is consistent with the way game designers discuss/describe genres by example [19].

While the story space of our example scenario refers to a simple medieval fantasy genre, our conceptual modelling method should be able to cope with an ample variety of genres of higher complexity. The structure of each story space thus specified is determined by the observed event relations. From an informal viewpoint (cf. Figure 1), events can be seen as nodes and syntagmatic, paradigmatic relations as connecting edges drawn over a plane, while meronymic relations appear as projections of events into event sequences over another plane. Antithetic relations between nodes can be seen as specifying constraints crosscutting the story space. Recall that paths formed by syntagmatic relations correspond to plots, whereas paradigmatic relations indicate alternatives - whose choice may entail, as signaled by antithetic relations, the exclusion of certain other alternatives branching from subsequent nodes.

Supporting the identified relations also brings extra benefits. Plot libraries can be organized as combined is-a and part-of hierarchies, which is a convenient way to deal with plots by taking advantage of the similarity or analogy among situations. While the part-of links result directly from meronymic relations, denoting in consequence composition / decomposition, the is-a links express the notion of generalization / specialization. If two or more events stand in a paradigmatic relation, their main effects should coincide, although they may differ with respect to less important side-effects. For instance, abduction and elopement can be seen as alternative forms of villainy. This justifies the introduction of a new event, which might be named villainy (or some more specific term), and its placement in a hierarchy above the other two one-event plots, i.e. it generalizes both. These relations contribute to the hierarchical structure of the library and, once it is constructed, its component patterns can then be reused during the plot composition process to help create new plots. Because our approach employs plangeneration/plan-recognition while composing a plot, we regard "typical plots" in our context as synonymous to typical plans. These relations also bring a particularly convenient way to deal with entire plots, helping visualize their dramatic structure.

Finally, let us recall that we have addressed the fabula level only, where one simply indicates which events should be included in the plots. One especially complex problem to be faced at the next level - story/narration, where the concern is how to tell the events - is how to properly convert the events into forms adequate for dramatization. 


\section{LogTell-R}

To implement this model we extended LOGTELL's interaction modes and dynamic schema level, i.e. the set of operations that can transform the storyworld, to take into consideration the four relations previously presented. As mentioned before, the Plot Manager module originally dealt only with the syntagmatic and paradigmatic axes. The changes introduced in our new prototype now allow meronymic relations by introducing complex operations, and antithetic relations, by employing motifs.

When dealing with support for antithetic relations, since we emphasize interactive composition, we considered the possibility of user interventions that can result in some sort of discontinuity being produced. If we see a disruption not as a discontinuity in one context, but as an attempt to put together two originally incompatible contexts, then the notion of blending [3] immediately comes to mind, as the technique or artisanship of conciliating the pending conflicts, which often requires a great deal of creativity. Incompatibilities between events - which we have been characterizing in terms of antithetic event relations - are usually induced by some factor involving the current value of a property or the beliefs of a character. A genre can in general be seen as a contract between author and audience [7] - in the sense that the audience knows what to expect - even if some event almost crosses the borderline of plausibility. Fortunately, blending can be achieved in a convenient way by resorting to folktale motifs [18], which often encode ingenious solutions to contradictions or dead-ends in a tale, while seeming to fit with the conventions of their encompassing genres. In order to support this mediation, LogTell-R permits the insertion of a motif at an appropriate position in the plot. This motif must come from a pre-defined set, and takes the form of a special (pseudo-)operation. Motifs are associated with either a goal specification or a set of post-conditions. In our proof-ofconcept, we selected a set of genre-suitable motifs (e.g. life token - that allows to do without the unrealistic assumption that characters are omniscient). Motifs also act as dramatization tools, providing the audience a convincing explanation of why certain events must ensue. In our prototype, a motif is associated with a script defining the behaviour of the involved characters when dramatizing it.

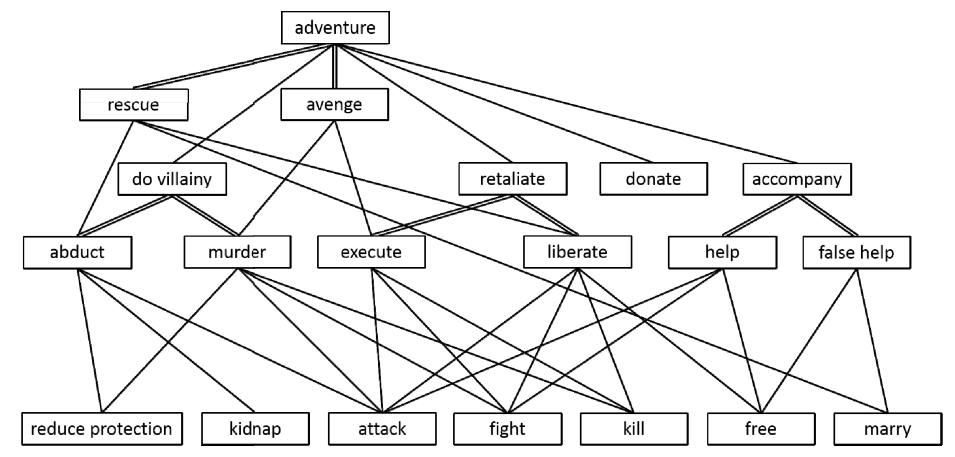

Fig. 2. Hierarchy of typical plans used in most of our experiments 
Support for complex operations in plan recognition and the use of the hierarchy of typical plans can be seen as a complement to generation. The benefits of the plan library structure itself are twofold: it helps better visualize story structure, showing how to adequately chain events into a narrative scheme (like the three-parts classic structure [10]); and it helps in more easily understanding and analyzing the space of stories that can be generated. In LogTell-R, plot generation still happens in a stepwise fashion. A new way of choosing alternatives is enabled by selecting events and asking the plan-recognizer to retrieve generalizations containing them. Exploiting the meronymic axis at different levels of detail, plausible alternatives may be located by essentially walking up and down over the hierarchy. Instead of asking the system for suggestions, the user may also simply find it helpful to visually inspect the library of typical plans associated with the genre (Figure 2), and use it as inspiration to guide the composition of a plot.

As our approach is centered on the concept of hierarchies of typical plans and given that conceptual models tend to remain too far removed from system implementations, we narrow this gap with the help of an intermediate logical design stage. The approach utilized is a plot manipulation algebra (PMA) [9] for the creation of the hierarchy of typical plans. An added benefit of using PMA (supported by the planner to check pre- and post- conditions of operations) in the knowledge base editor is that one can more easily experiment with different ways to chain the events, and use this knowledge to revise the specification so as to improve the system's story space. Starting with the set of operations relevant for the genre (e.g. abduct, murder, liberate), algebra operands are applied to define the necessary relations between events (e.g. generalizations or placement of constraints on undesired event combinations). At each step, the user can visualize how the typical plan hierarchy is "grown".

\section{Concluding Remarks}

Few approaches to IS address the problem of what is a proper story space or the characteristics of events pertaining to a plot, preferring to focus on more controlled environments and stories. We feel that more generative approaches are necessary for the field to become truly successful. It is important to stress, however, that we do not propose to achieve a fully immersive experience. We endeavour, instead, to explore the possibilities of generating a large variety of coherent and interesting stories through the use of plan-generation and recognition over the conceptual model of a genre.

Our approach targets sound methods to organize and combine events, in ways that may confer enough dramatic power to the generated narratives. By extending our conceptual model, we augment its expressiveness and provide tools to prospective authors intent on creating and telling stories. Although the process of plot composition and adaptation could surely be enriched beyond what is presented here, our system provides a sound basis to treat genres that exhibit a good degree of regularity. 
Having authorial tools make the complexities of the model transparent to authors is a major goal, primarily to reduce the burden on authors using the system, but also to guide them in the creation of interesting stories. Creating an interactive environment that behaves as expected is a tiresome task, as it is performed mostly by trial and error.

\section{References}

1. Bal, M.: Narratology. University of Toronto Press, Toronto (2002)

2. Burke, K.: A Grammar of Motives. University of California Press, Oakland (1969)

3. Casanova, M.A., et al.: Generalization and Blending in the Generation of EntityRelationship Schemas by Analogy. In: Proceedings of ICEIS, Spain (2008)

4. Ciarlini, A.E.M., Pozzer, C.T., Furtado, A.L., Feijó, B.: A Logic-based Tool for Interactive Generation and Dramatization of Stories. In: Proceedings of ACE 2005, Spain (2005)

5. Culler, J.: On Deconstruction. Cornell University Press, Ithaca (1983)

6. Ciarlini, A.E.M., Barbosa, S.D.J., Casanova, M.A., Furtado, A.L.: Event Relations in PlanBased Plot Composition. ACM Computers in Entertainment 7, 4 (2009)

7. Glassner, A.: Interactive Storytelling: Techniques for 21 st Century Fiction (2004)

8. Karlsson, B., Ciarlini, A., Feijó, B., Furtado, A.L.: Applying the Plan-Recognition/PlanGeneration Paradigm to Interactive Storytelling. In: ICAPS 2006 Workshop on AI Planning for Computer Games and Synthetic Characters, Lake District, UK (2006)

9. Karlsson, B., Barbosa, S.D.J., Furtado, A.L., Casanova, M.A.: A Plot-Manipulation Algebra to Support Digital Storytelling. In: Natkin, S., Dupire, J. (eds.) ICEC 2009. LNCS, vol. 5709, pp. 132-144. Springer, Heidelberg (2009)

10. Karlsson, B.: A Model and an Interactive System for Plot Composition and Adaptation, based on Plan Recognition and Plan Generation. PhD Thesis, PUC-Rio, Brazil (2010)

11. Lang, R.R.: A Declarative Model for Simple Narratives. In: AAAI Fall Symposium (1999)

12. Magerko, B.: A Comparative Analysis of Story Representations for Interactive Narrative Systems. In: Proceedings of AIIDE 2007, USA (2007)

13. Mateas, M., Stern, A.: Façade: An Experiment in Building a Fully-Realized Interactive Drama. In: Game Developers Conference, pp. 4-8 (2003)

14. Nakasone, A., Ishizuka, M.: ISRST: An interest based storytelling model using rhetorical relations. In: Hui, K.-C., Pan, Z., Chung, R.C.-K., Wang, C.C.L., Jin, X., Göbel, S., Li, E.C.-L. (eds.) Edutainment 2007. LNCS, vol. 4469, pp. 324-335. Springer, Heidelberg (2007)

15. Pinhanez, C.: Interval Scripts: A Design Paradigm for Story-Based Interactive Systems. In: Proceedings of CHI 1997, Atlanta, USA (1997)

16. Propp, V.: Morphology of the Folktale. S. Laurence (trans.). Univ. of Texas Press (1968)

17. Schank, R.C., Colby, K.: Computer Models of Thought and Language. Freeman (1973)

18. Thompson, S.: Motif-Index of Folk-Literature. Indiana University Press, USA (1989)

19. Wallis, J.: Making Games that Make Stories. In: Second Person. MIT Press (2007) 